On the role of vibrational energy in the activated dissociative chemisorption of methane on tungsten and rhodium

C. T. Rettner, H. E. Pfnür, and D. J. Auerbach

Citation: The Journal of Chemical Physics 84, 4163 (1986);

View online: https://doi.org/10.1063/1.450035

View Table of Contents: http://aip.scitation.org/toc/jcp/84/8

Published by the American Institute of Physics

Articles you may be interested in

Dynamics of activated chemisorption: Methane on rhodium

The Journal of Chemical Physics 62, 4672 (1975); 10.1063/1.430415

AP| $\begin{aligned} & \text { The Journal of } \\ & \text { Chemical Physics }\end{aligned}$

PERSPECTIVES 


\title{
On the role of vibrational energy in the activated dissociative chemisorption of methane on tungsten and rhodium
}

\author{
C. T. Rettner, H. E. Pfnür, ${ }^{\text {a) }}$ and D. J. Auerbach \\ IBM Research Laboratory, San Jose, California 95136
}

(Received 12 September 1985; accepted 28 December 1985)

\begin{abstract}
The vibrational energy dependence of the dissociative chemisorption probability of $\mathrm{CH}_{4}$ on $\mathrm{W}(110)$ is investigated with the use of a seeded supersonic molecular beam. By variation of the beam source temperature and seed gas mixture the degree of vibrational excitation of the incident $\mathrm{CH}_{4}$ molecules can be varied while the incident kinetic energy is held constant. The results are consistent with a model in which all vibrational modes are equally effective and vibrational energy and translational energy are approximately equivalent in promoting this highly activated process. Previous unsuccessful attempts to promote $\mathrm{CH}_{4}$ chemisorption on rhodium via vibrational excitation are consistent with our findings; we are also able to account for previous observations of enhancement of dissociative chemisorption in heated effusive beam experiments.
\end{abstract}

\section{INTRODUCTION}

The dissociative chemisorption probability is usually quite high for small molecules on clean metal surfaces. Notable exceptions are the chemisorption of nitrogen and of saturated hydrocarbons, which often adsorb with probabilities of $\leqslant 10^{-3}$. Here chemisorption appears to be an activated process, with a potential energy barrier limiting the rate of dissociation. In recent studies, ${ }^{1,2}$ we have shown that for chemisorption on W(110), this barrier can be efficiently overcome by providing the reactants with translational energy. For $\mathrm{N}_{2}$, the dissociative chemisorption probability in the limit of zero coverage, $S_{0}$, was found to increase by a factor of more than 100 for a $1 \mathrm{eV}$ increase in translational energy, ${ }^{1}$ while for $\mathrm{CH}_{4}, \mathrm{a} \sim 10^{5}$ increase was observed. ${ }^{2}$ Qualitatively similar activation has been reported previously for the $\mathrm{H}_{2} / \mathrm{Cu}$ system, ${ }^{3}$ although in that case only a factor of $\sim 5$ increase in $S_{0}$ was observed with increasing translational energy.

During the course of the $\mathrm{CH}_{4} / \mathrm{W}(110)$ study, we observed that beams of similar translational energy, but originating from nozzles of differing temperatures, displayed substantially different sticking probabilities. Here we present an analysis and discussion of these findings and show that our observations are consistent with a model based on the equal participation of all vibrational modes and a rough equivalence of vibrational and translational energy in their ability to enhance the dissociative chemisorption of $\mathrm{CH}_{4}$ on $\mathrm{W}(110)$. We also reexamine three previous studies ${ }^{4-6}$ concerned with the enhancement of $\mathrm{CH}_{4}$ chemisorption via vibrational energy. We are able to resolve apparent contradictions between these studies and to show that the previous experimental results are all consistent with our model.

\section{EXPERIMENTAL}

The apparatus and experimental techniques have been described previously. ${ }^{1,2,7} \mathrm{~A}$ supersonic beam of $\mathrm{CH}_{4}$ is inci-

\footnotetext{
a) Present address: Fakultät für Physik der Technischen Universität München, Physik-Department E20, 8046 Garching b. München, West Germany.
}

dent on a W(110) crystal mounted in a UHV chamber on a manipulator which permits accurate control of the incidence angle and provides for $e$-beam heating and liquid nitrogen cooling. Temperature, monitored with a thermocouple spot welded to the back of the sample, is controlled by a feedback system interfaced to a minicomputer (IBM, Series 1).

Beam energies are varied by changing the nozzle temperature and by seeding the $\mathrm{CH}_{4}$ in $\mathrm{H}_{2}, \mathrm{He}$, or Ar. These energies are determined from flight times from a high-speed chopper to a differentially pumped rotatable mass spectrometer. Typical energy spreads of $\sim 15 \%$ of the incidence energy (FWHM) are obtained with a reservoir pressure of 30 to 50 psi behind a $100 \mu \mathrm{m}$ diameter nozzle. $S_{0}$ values presented here are determined from the initial slopes of coverage vs exposure curves. Exposures are varied by controlling the dose time of a beam whose flux can be determined from its area and the partial pressure rise produced on the system. Surface converages are determined from the following (computer-controlled) procedure. After exposure to the methane beam, the sample is exposed to a saturation dose ( $~\left(10\right.$ monolayers) of $\mathrm{O}_{2}$ from an auxiliary beam at a surface temperature of $600 \mathrm{~K}$. The sample temperature is then ramped while recording the partial pressure of $\mathrm{CO}$ in the system on the same residual gas analyzer, RGA (Vacuum Generators, model SX200) used to determine the beam flux. The amount of carbon left on the surface after the methane dissociates is then obtained from the area under the resulting flash desorption spectrum which peaks at about $1100 \mathrm{~K}$. (Note that the hydrogen product of this reaction desorbs above $\sim 500 \mathrm{~K}^{8}$ ) This measurement requires knowledge of the $\mathrm{CO}$ pumping speed, which is measured at the end of a run, and the relative sensitivity of the RGA to $\mathrm{CO}$ and $\mathrm{CH}_{4}$, determined in separate experiments with pure $\mathrm{CO}$ and $\mathrm{CH}_{4}$ beams referenced to pressures measured on an ionization gauge. This method was cross checked for high-energy measurements (with $S_{0}$ values $\gtrsim 5 \times 10^{-2}$ ) by using a direct method involving measurement of the fraction of the beam reflected by the surface (following the approach of King and Wells ${ }^{9}$ ). In addition, Auger electron spectroscopy has been employed to measure surface coverages directly.

Care was taken in these measurements to exclude con- 
TABLE I. Effect of nozzle temperature on initial sticking probabilities of $\mathrm{CH}_{4}$ on W(110).

\begin{tabular}{lccc}
\hline \hline $\begin{array}{c}\text { Trans. energy } \\
(\mathrm{eV})\end{array}$ & $\begin{array}{c}\text { Surface temp. } \\
(\mathrm{K})\end{array}$ & $\begin{array}{c}\text { Nozzle temp. } \\
(\mathrm{K})\end{array}$ & $S_{0}$ \\
\hline $0.11 \pm 0.01^{\mathrm{a}}$ & 800 & 770 & $2.5 \pm 1.0 \times 10^{-5}$ \\
$0.11 \pm 0.01$ & 800 & 340 & $5.0 \pm 2.0 \times 10^{-6}$ \\
$0.23 \pm 0.02$ & 800 & 720 & $4.0 \pm 2.0 \times 10^{-5}$ \\
$0.23 \pm 0.02$ & 800 & 305 & $7.0 \pm 5.0 \times 10^{-6}$ \\
\hline
\end{tabular}

Error bars reported here refer to approximate $95 \%$ confidence limits.

tributions to the apparent sticking probability due to chemistry in the beam source promoted by the higher source temperatures. Two control experiments were carried out. In the first, a section of stainless steel tubing identical to that used to form the source was placed in the gas inlet line $30 \mathrm{~cm}$ upstream of the nozzle and heated to $770 \mathrm{~K}$ while keeping the source at $340 \mathrm{~K}$. Thus the gas mixture had to pass through this section before reaching the nozzle. In flowing through this tube, the gas mixture should experience similar heating and residence times as for the $770 \mathrm{~K}$ source experiment and is at the same pressure. Thus we would expect any chemical transformations to be reproduced, i.e., formation of higher hydrocarbons, etc., while any thermal excitations should be relaxed prior to reaching the nozzle. Sticking probabilities measured with this arrangement were found to be slightly higher, by a factor of $\leqslant 2$, than for the "genuine" 340 $\mathrm{K}$ measurement. This contribution (which amounts to about $20 \%$ of the observed signal) has been subtracted from the data reported. In a second control experiment, the apparent sticking probability was measured for a $770 \mathrm{~K}$ source with a pure Ar beam. Here we would observe any contributions due to carbonaceous material entering the beam after desorbing from source components. This effect amounted to $<50 \%$ of the cold source probability and is within the experimental uncertainties. We note that such contributions serve only to decrease the apparent effect of vibrational excitation on the dissociative chemisorption probability.

\section{RESULTS}

Table I gives the results of two experiments in which we sought to determine the effect of nozzle temperature on the effective initial sticking probability of $\mathrm{CH}_{4}$ on $\mathrm{W}(110)$. Measurements at 0.11 and $0.23 \mathrm{eV}$ both show about a fivefold increase in sticking probability on increasing the nozzle temperature from $\sim 300$ to $\sim 700 \mathrm{~K}$. Here the translational energies were adjusted by controlling the seeding ratio in $\mathrm{Ar} / \mathrm{He}$ mixtures. Time-of-flight measurements on these beams gave $\Delta E / E$ ratios of $\leqslant 0.15$, and the observed effects are clearly not due to broadening of the translational distributions at higher source temperatures. Effects due to rotational excitation cannot be ruled out on the basis of the current measurements alone, since we have no specific knowledge of the degree of rotational relaxation. However, rotational relaxation must be expected to be considerably greater than vibrational, ${ }^{10}$ since relaxation tends to "freeze" as the relevant energy level spacing approaches $k T$. Thus we expect relatively little vibrational relaxation for methane which has vibrational spacings of over $1000 \mathrm{~cm}$, but can anticipate extensive rotational relaxation, as the rotational constant is only $5 \mathrm{~cm}$. Comparison with previous studies of relaxation in beams of $\mathrm{N}_{2},{ }^{10,11}$ and with our own experience of relaxation in beams of NO and CO, suggest that the rotational energy should be less than $10 \%$ of that at the nozzle, or $\leqslant 80 \mathrm{~K}$, for our conditions. Thus it is highly unlikely that the observed effects are influenced strongly by changes in levels of rotational excitation, although some rotational contribution cannot be ruled out. Furthermore, it is difficult to picture how rotational energy could be efficiently channeled into the reaction coordinate, which is presumably why Winters ${ }^{8}$ did not consider its effect, despite the fact the rotational and vibrational temperatures were most likely equal for his conditions. We conclude that the observed changes in $S_{0}$ result predominantly from the presence of vibrationally excited species in the beam.

\section{ANALYSIS AND DISCUSSION}

The dissociative chemisorption of $\mathrm{CH}_{4}$ on $\mathrm{W}(110)$ is a highly activated process, with the initial sticking probability depending sensitively on both the incidence energy, $E_{i}$, and angle, $\theta_{i}$. We have shown ${ }^{2}$ that over the range 0.1 to $1.1 \mathrm{eV}$ the initial chemisorption probability increases exponentially with the "normal kinetic energy," $E_{n}$, where $E_{n}=E_{i}$ $\cos ^{2} \theta_{i}$. This behavior was found to be consistent with a

TABLE II. Sticking probabilities for vibrationally excited $\mathrm{CH}_{4}$.

\begin{tabular}{cccccccc}
\hline $\begin{array}{c}\text { Mode } \\
v_{i}\end{array}$ & $\begin{array}{c}\text { Frequency } \\
\left(\mathrm{cm}^{-1}\right)\end{array}$ & Degeneracy & $\begin{array}{c}\mathrm{Ni} / \mathrm{N} \\
\text { at } 770 \mathrm{~K}\end{array}$ & $S_{0}^{a}\left(v_{i}\right)^{\mathrm{a}}$ & $\begin{array}{c}\Delta \ln S_{0} \\
\Delta E_{\text {vib }} \\
\left(\mathrm{eV}^{-1}\right)\end{array}$ & $S_{0}^{b}\left(v_{i}\right)^{\mathrm{b}}$ & $\begin{array}{c}\text { Relative }^{\mathrm{c}} \\
\text { contribution } \\
(\%)\end{array}$ \\
\hline$v_{1}$ & 2914 & 1 & $2.8 \times 10^{-3}$ & $7.1 \times 10^{-3}$ & 20.1 & $4.4 \times 10^{-4}$ & 5 \\
$v_{2}$ & 1526 & 2 & $7.6 \times 10^{-2}$ & $2.6 \times 10^{-4}$ & 20.9 & $5.2 \times 10^{-5}$ & 16 \\
$v_{3}$ & 3020 & 3 & $6.8 \times 10^{-3}$ & $2.9 \times 10^{-3}$ & 17.0 & $5.2 \times 10^{-4}$ & 14 \\
$v_{4}$ & 1306 & 3 & $1.7 \times 10^{-1}$ & $1.1 \times 10^{-4}$ & 19.1 & $3.7 \times 10^{-5}$ & 25 \\
$2 v_{2}$ & 3010 & 2 & $4.7 \times 10^{-3}$ & $4.2 \times 10^{-4}$ & 18.1 & $5.1 \times 10^{-4}$ & 10 \\
$2 v_{4}$ & 2600 & 3 & $1.5 \times 10^{-2}$ & $1.3 \times 10^{-4}$ & 17.3 & $2.7 \times 10^{-4}$ & 16 \\
Vibrational & Ground & States & $7.2 \times 10^{-1}$ & $\ldots$ & $\ldots$ & $5 \times 10^{-6}$ & 14 \\
\hline \hline
\end{tabular}

- Based on the assumption that the ith state is entirely reponsible for the observed increase in the sticking probability. Obtained by dividing the observed increase in sticking probability of $2.0 \times 10^{-5}$ by $\mathrm{Ni} / \mathrm{N}$.

${ }^{b}$ Based on the assumption that all vibrational states contribute to the observed increase in the sticking probability, in proportion to their energy, assuming: $S_{0}\left(v_{i}\right)=5 \times 10^{-6} \times \exp \left[12.4 \times E_{\mathrm{vib}}(\mathrm{eV})\right]$.

${ }^{c}=100 \times(\mathrm{Ni} / \mathrm{N}) \times S_{0}^{b}\left(v_{i}\right) / 2.5 \times 10^{-5}$. 
model in which a hydrogen atom tunnels through a barrier in the reaction coordinate. ${ }^{2}$ We obtained the semiempirical result

$$
S_{0} \simeq \alpha \exp \left(\beta E_{n}\right),
$$

where $\alpha \sim 2.0 \times 10^{-6}$, and $\beta \sim 12.5 \mathrm{eV}^{-1}$. These values are consistent with tunneling through a parabolic barrier of height $1.1 \mathrm{eV}$ and a half-width $0.25 \AA .^{2}$ In analyzing the effect of vibrational energy on the sticking probability, we will assume a similar exponential dependence. A comparison of the $\beta$ values for translation and for vibration may then reveal the relative efficacy of these two modes of activation.

There are two limiting cases for the variation of $S_{0}$ with vibrational energy, $E_{\text {vib }}$. In one extreme, we can attribute the observed effects to a single vibrational state that couples strongly to the reaction coordinate. Alternatively we can assume that all vibrational modes are equally efficacious in promoting dissociation. These two possiblities lead to two different sets of values for the sticking probabilities associated with each vibrational state. Assuming a single state to be responsible for the observed effect gives an initial sticking probability for $\mathrm{CH}_{4}$ in that state of $S_{0}^{a}\left(\nu_{i}\right)$, which will be considerably larger than the value $S_{0}^{b}\left(v_{i}\right)$, obtained assuming that all states contribute to the observed increase in $S_{0}$. Table II displays the fraction of vibrationally excited $\mathrm{CH}_{4}$ states of $770 \mathrm{~K}$, together with an estimate for the calculated sticking probabilites, $S_{0}^{a}\left(\nu_{i}\right)$, associated with each state, assuming that state to be solely responsible for the observed increase. Also shown are values of the effective efficacy values, $\beta$, associated with these sticking probabilities, where we define this efficacy as the slope

$$
\beta_{\text {vib }}=\Delta \ln S_{0} / \Delta E_{\mathrm{vib}}\left(\nu_{i}\right) \text {. }
$$

These values should be compared with the value of

$$
\beta_{\text {trans }}=\Delta \ln S_{0} / \Delta E_{\text {trans }}\left(E_{n}\right),
$$

where $\beta_{\text {trans }} \simeq 12.5 \mathrm{eV}^{-1}$, as obtained from the translational energy dependence of $S_{0}$ as described above. Clearly they are all considerably larger than this value. Thus a simplistic analysis, assuming a single vibrational mode is responsible for the enhancement in $S_{0}$, would indicate that placing energy in such a mode is more efficient than in translation. One way to quantify these differences is to consider the ratios, $\beta_{\text {vib }} / \beta_{\text {trans }}$. Such ratios indicate the relative fractions of the vibrational and translational energy that are available to overcoming the barrier in the reaction coordinate. We see that in no case is this ratio more than a factor of 2 . Nevertheless, because of the steep dependence of $S_{0}$ on energy in this system, small increases in efficacy may result in large increases in the sticking coefficient. Specifically,

$$
S_{0}\left(E_{\mathrm{vib}}\right) / S_{0}\left(E_{\text {trans }}\right)=\exp \left[\left(\beta_{\text {vib }}-\beta_{\text {trans }}\right) E_{n}\right] \text {. }
$$

Thus $0.16 \mathrm{eV}$ placed in the $v_{4}$ mode may lead to a sticking coefficient $\sim 3$ times larger than for the same energy in translation. However, note that this is very much a limitingcase result, since it was obtained assuming zero contribution from all other modes.

If we assume instead that all vibrational modes are equally efficacious, we arrive at almost the opposite conclusion. In this case the slope

$$
\beta_{\text {vib }}=\Delta \ln S_{0} / \Delta E\left(E_{\text {vib }}\right) \simeq 12.4 \mathrm{eV}^{-1}
$$

is essentially identical to that for translation. Combining this result with the translational energy result leads to an overall expression for $S_{0}$ of the form

$$
S_{0} \simeq 2.0 \times 10^{-6} \exp \left[12.5\left(E_{\text {trans }}+E_{\text {vib }}\right)\right] \text {. }
$$

The observed increase in $S_{0}$ from $5 \times 10^{-6}$ for a source temperature of $340 \mathrm{~K}$ to $2.5 \times 10^{-5}$ for $770 \mathrm{~K}$ (with $E_{\text {trans }}$ $=0.11 \mathrm{eV}$ in both cases) then arises from a large number of roughly equal contributions from a number of $\mathrm{CH}_{4}$ vibrational modes, as shown in Table II. These contributions are also listed in Table II, together with the corresponding sticking probabilities, $S_{0}^{b}\left(v_{i}\right)$, for the individual modes, which refer to a methane molecule having $0.11 \mathrm{eV}$ of translational energy. Analysis of the $0.23 \mathrm{eV}$ data points leads to similar conclusions.

No account has been taken in this analysis for possible vibrational relaxation either in the beam expansion or at the surface prior to reaction. In this respect, the present results represent only a lower limit to the sticking probabilities associated with vibrationally excited $\mathrm{CH}_{4}$ molecules. However, relaxation in the beam should be no more than $50 \%$ for the low energy modes, ${ }^{12}$ and substantially less for the higher energy states. Moreover, while relaxation at the surface might contribute if the reaction were to proceed via a precursor mechanism, whereby the molecule can accommodate to the surface, the dramatic translational energy dependence observed strongly argues for a direct reaction mechanism, making such relaxation rather unlikely. The contribution to $S_{0}$ from other hydrocarbon species in the beam has been allowed for as discussed in Sec. II and amounts to $\sim 20 \%$ of what we consider to be the true signal due to dissociation of vibrationally excited methane.

The present results indicate that vibrational and translational energy are roughly equivalent in helping to overcome the barrier to chemisorption of $\mathrm{a} \mathrm{CH}_{4}$ molecule on $\mathrm{W}(110)$. Such behavior would result if the surface collision is associated with substantial scrambling of vibrational and translational energy. However, since we observe fairly precise normal energy scaling ${ }^{2}$ in this system, the scrambling process cannot be such as to access parallel momentum.

Our results are consistent with previous unsuccessful attempts to observe vibrational enhancement of the sticking probability of $\mathrm{CH}_{4}$ on rhodium surfaces. ${ }^{5,6} \mathrm{Here}$ a $\mathrm{He} / \mathrm{Ne}$ laser was employed to pump individual $\mathrm{CH}_{4}$ vibrational modes. Brass et al. ${ }^{5}$ reported that $S_{0}$ for $\mathrm{CH}_{4}$ excited to the $v_{3}$ level is $<1 \times 10^{-4}$ while that for the $2 v_{3}$ level is estimated to be $<7.0 \times 10^{-2}$. For a $303 \mathrm{~K}$ gas they estimate $S_{0} \sim 10^{-7}$. In a similar experiment on $\mathrm{Rh}(111)$, Yates et al. ${ }^{6}$ obtained sticking probabilities for excited $\nu_{3}$ and $2 v_{4} \mathrm{CH}_{4}$ species of $\leqslant 5 \times 10^{-5}$. If a similar mechanism as for the $\mathrm{CH}_{4} / \mathrm{W}(110)$ system were to apply to the $\mathrm{CH}_{4} / \mathrm{Rh}$ system, we would predict sticking probabilities of

$$
S_{0} \sim 1 \times 10^{-7} \exp \left[12.4 \times E_{\mathrm{vib}}\left(\nu_{i}\right)\right],
$$
giving $1.0 \times 10^{-3}, 1.0 \times 10^{-5}$, and $5.0 \times 10^{-6}$, for the $2 v_{3}, v_{3}$, and $2 v_{4}$ modes, respectively, in keeping with these observations.

These results appear to contradict an earlier study of Steward and Ehrlich in which heating an effusive methane 


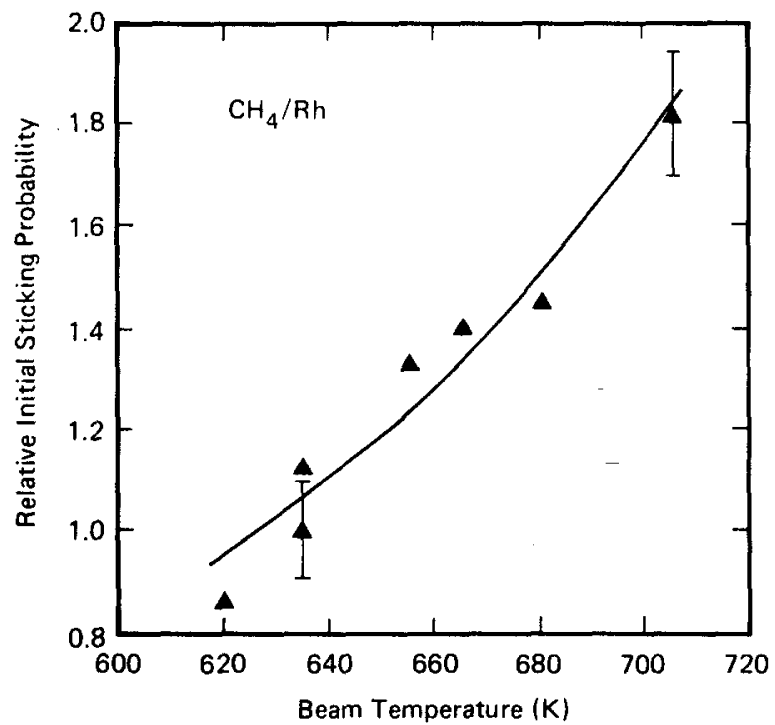

FIG. 1. Initial sticking probability as a function of source temperature for an effusive $\mathrm{CH}_{4}$ beam incident on rhodium (data points of Fig. 7, Ref. 4). The line indicates the fit described in the text.

source up to $\sim 700 \mathrm{~K}$ yielded a large increase in sticking probability which was interpreted as being due to the increased vibrational excitation of the molecules in the hot beam. Yates et al. suggested that $\mathrm{H}_{2}$ formed in the $\mathrm{CH}_{4}$ beam source at these temperatures may have interfered with the sticking measurements. While this may be one possibility, we believe that this data can be accounted for without invoking a high chemisorption probability for vibrationally excited molecules. Stewart et al. based their hypothesis of vibrational enhancement, rather than translational enhancement, entirely on the differing sticking probabilities observed for $\mathrm{CH}_{4}$ compared to $\mathrm{CD}_{4}$. They argued that since both molecules must have the same translational energy distribution, the observed behavior could not be attributable to the effect of translational energy on the chemisorption probability. In our interpretation, this difference is a result of the higher tunneling probability for the $\mathrm{CH}_{4}$ case, as observed previously by us $^{2}$ and by Winters. ${ }^{8}$ The increase in sticking probability with increasing effusive beam source temperature then arises at least in part due to an increase in the translational energy of the beam.

As a starting point, we assume that the observed effect is entirely due to increasing the translational energy of the beam. The solid triangles in Fig. 1 show the data points taken from Ref. 4, while the solid line was obtained by assuming that the sticking probability increases as $S_{0} \propto \exp \left(\beta E_{\text {trans }}\right)$. Here we have convoluted over a Boltzmann distribution of translational energies for each beam temperature and carried out a nonlinear least squares fit to the experimental points. The resulting fit yielded $\beta=12.7 \mathrm{eV}^{-1}$, which is remarkably similar to the value of $12.5 \mathrm{eV}^{-1}$ found for the $\mathrm{CH}_{4} / \mathrm{W}(110)$ case, as discussed above. Thus these data can be accounted for without any consideration of vibrational energy effects, and as such cannot be used as evidence for such. However, the above analysis is clearly overly simplistic, since vibrational energy may very well increase the dissociation probability. Assuming equal efficacy for translational and vibrational energies leads to a virtually identical fit to that shown in Fig. 1. In this case we have calculated the contributions of the six lowest excited vibrational states, assuming

$$
S_{0}=\alpha \times \exp \left\{\beta \times\left[E_{\text {trans }}+E_{\text {vib }}\left(v_{i}\right)\right]\right\} .
$$

By weighting each state according to its Boltzmann population at the beam temperature and convoluting each over the appropriate translational energy distribution we have calculated values of $S_{0}$ which were fit to the observed data to again yield $\alpha$ and $\beta$. In this case we obtain $\beta=11.3 \mathrm{eV}^{-1}$. Here we find that the presence of vibrationally excited species in the beam causes the sticking probability to be about twice what it would be for a vibrationless system. Table III shows a breakdown of the contributions for a temperature of $705 \mathrm{~K}$. Including still higher vibrational states in the analysis will change these numbers by only a few percent. The relative sticking probabilities listed here correspond to molecules with a Boltzmann distribution of translational energies in a specific vibrational state. These values are referenced to an overall average value of 1.82 for this beam temperature.

In considering the dissociative chemisorption of methane on a heated tungsten wire, Winters ${ }^{8}$ assumes that chemisorption proceeds via vibrationally excited states. These states are thought to be populated after the methane comes to equilibrium at the surface temperature. While this may indeed be so, the experimental data does not in itself reveal any information about the relative role of the various degrees of freedom. The data yield only a phenomenological activation energy and are equally compatible with assignment of

TABLE III. Sticking probabilities for $\mathrm{CH}_{4}$ on rhodium for a $705 \mathrm{~K}$ effusive beam."

\begin{tabular}{|c|c|c|c|c|c|}
\hline $\begin{array}{c}\text { Mode } \\
v_{i}\end{array}$ & $\begin{array}{c}\text { Frequency } \\
\left(\mathrm{cm}^{-1}\right)\end{array}$ & Degeneracy & $\begin{array}{c}\mathrm{Ni} / \mathrm{N} \\
\text { at } 705 \mathrm{~K}\end{array}$ & $\mathrm{~S}_{0}\left(v_{i}\right)^{b}$ & $\begin{array}{c}\text { Contribution } \\
\text { to } S_{0}(\%)\end{array}$ \\
\hline$v_{1}$ & 2914 & 1 & $1.9 \times 10^{-3}$ & 56.0 & 5.8 \\
\hline$v_{2}$ & 1526 & 2 & $3.2 \times 10^{-2}$ & 8.0 & 14.2 \\
\hline$v_{3}$ & 3020 & 3 & $1.5 \times 10^{-3}$ & 64.4 & 5.4 \\
\hline$v_{4}$ & 1306 & 3 & $5.1 \times 10^{-2}$ & 5.9 & 16.3 \\
\hline $2 v_{2}$ & 3010 & 2 & $1.6 \times 10^{-3}$ & 63.5 & 5.5 \\
\hline $2 v_{4}$ & 2600 & 3 & $3.6 \times 10^{-3}$ & 35.8 & 7.1 \\
\hline Vibrational & Ground & States & $9.1 \times 10^{-1}$ & 0.94 & 47.0 \\
\hline
\end{tabular}

"Based on a fit to the experimental data of Ref. 4, assuming that translation and vibration are equal in their ability to promote dissociative chemisorption (see the text).

These sticking probabilities are relative values only, normalized to an average value of 1.82 for this beam temperature. 
the observed effects to translational or rotational motions. It is known, however, that the various deuterated analogs behave differently here, which led Winters to propose the quantum tunneling model for dissociative chemisorption in this system. ${ }^{8}$ Thus any proposed dynamical mechanism must allow for tunneling of hydrogen or deuterium along the reaction coordinate. This implies only that passage along the reaction coordinate is accompanied by breaking of a carbonhydrogen bond, and gives no indication per se as to which form of energy most strongly couples to this coordinate.

\section{SUMMARY AND CONCLUSIONS}

We have shown that vibrational excitation enhances the initial chemisorption probability of $\mathrm{CH}_{4}$ on $\mathrm{W}(110)$, but that, at least on average, this enhancement is not significantly larger than for an equivalent amount of energy placed into translation. While it is possible that our results are infuenced by vibrational relaxation prior to dissociation or by the presence of spurious hydrocarbons in the molecular beam, these effects are considered to be too small to effect this conclusion. It is possible that individual vibrational modes contribute disproportionately to the observed average sticking probability, but in that case other modes would need to be less efficacious than translation in promoting reaction. These results are found to be consistent with previous unsuccessful attempts to promote chemisorption of methane on rhodium via laser excitation of specific vibrational modes. A previous report of an apparent strong vibrational energy effect has been reanalyzed and shown to be consistent with translational activation, with a possible equal contribution from vibrational energy.

The result that vibrational energy and translational energy are roughly equivalent in promoting reaction is intriguing. Such behavior could arise if the system were to pass through a negative ion precursor, as discussed by Gadzuk and Holloway. ${ }^{13}$ Here the molecule may rattle around on the surface prior to dissociation, during which time translational and vibrational energy may become substantially mixed before the system passes over the barrier to dissociation. This model has been proposed to account for the observed total-energy scaling in the $\mathrm{N}_{2} / \mathrm{W}(110)$ system. ${ }^{1,13}$ The fact that normal energy scaling is observed here implies that the mixing of energy associated with motions parallel and perpendicular to the surface must be slow compared to the lifetime of this negative ion state, while the mixing of normal and vibrational energy would have to be relatively fast on this time scale.

We thank J. E. Schlaegel and P. Alnot for valuable contributions to these experiments; many helpful discussions with H. F. Winters are gratefully acknowledged.

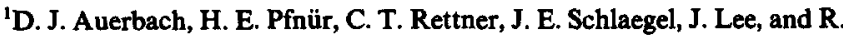
J. Madix, J. Chem. Phys. 81, 2515 ( 1984); H. E. Pfnür, C. T. Rettner, D. J. Auerbach, R. J. Madix and J. Lee, ibid. (to be submitted).

${ }^{2}$ C. T. Rettner, H. E. Pfnür, and D. J. Auerbach, Phys. Rev. Lett. 54, 2716 (1985).

${ }^{3}$ M. Balooch, M. J. Cardillo, D. R. Miller, and R. E. Stickney, Surf. Sci. 46, 358 (1974).

${ }^{4}$ C. N. Stewart and G. Ehrlich, J. Chem. Phys. 62, 4672 (1975)

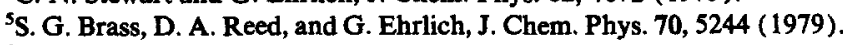

${ }^{6} \mathrm{~J}$. T. Yates, Jr., J. J. Zinck, S. Sheard, and W. H. Weinberg, J. Chem. Phys. 70, 2266 (1979).

${ }^{7}$ J. Lee, R. J. Madix, J. E. Schlaegel, and D. J. Auerbach, Surf. Sci. 143, 626 (1984).

${ }^{8}$ H. F. Winters, J. Chem. Phys. 62, 2454 (1975); 64, 3495 (1976).

'D. A. King and M. G. Wells, Surf. Sci. 29, 454 (1971).

${ }^{10} \mathrm{~J}$. J. Repetski and R. E. Mates, Phys. Fluids 14, 2605 (1971).

${ }^{11}$ S. Yamazaki, M. Taki, and Y. Fujitani, J. Chem. Phys. 74, 4476 (1981).

${ }^{12}$ T. A. Milne, J. E. Beachey, and F. T. Greene, J. Chem. Phys. 56, 3007 (1972).

${ }^{13} \mathrm{~J}$. W. Gadzuk and S. Holoway, Chem. Phys. Lett. 114, 314 (1985); S. Holoway and J. W. Gadzuk, J. Chem. Phys. 82, 5203 (1985). 